\title{
Carnosine inhibits carbonic anhydrase IX-mediated extracellular acidosis and suppresses growth of HeLa tumor xenografts
}

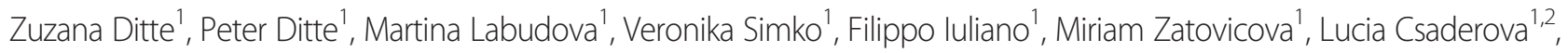
Silvia Pastorekova ${ }^{1}$ and Jaromir Pastorek ${ }^{1 *}$

\begin{abstract}
Background: Carbonic anhydrase IX (CA IX) is a transmembrane enzyme that is present in many types of solid tumors. Expression of CA IX is driven predominantly by the hypoxia-inducible factor (HIF) pathway and helps to maintain intracellular pH homeostasis under hypoxic conditions, resulting in acidification of the tumor microenvironment. Carnosine ( $\beta$-alanyl-L-histidine) is an anti-tumorigenic agent that inhibits the proliferation of cancer cells. In this study, we investigated the role of CA IX in carnosine-mediated antitumor activity and whether the underlying mechanism involves transcriptional and translational modulation of HIF-1a and CA IX and/or altered CA IX function.

Methods: The effect of carnosine was studied using two-dimensional cell monolayers of several cell lines with endogenous CA IX expression as well as Madin Darby canine kidney transfectants, three-dimensional HeLa spheroids, and an in vivo model of HeLa xenografts in nude mice. mRNA and protein expression and protein localization were analyzed by real-time PCR, western blot analysis, and immunofluorescence staining, respectively. Cell viability was measured by a flow cytometric assay. Expression of HIF-1a and CA IX in tumors was assessed by immunohistochemical staining. Real-time measurement of $\mathrm{pH}$ was performed using a sensor dish reader. Binding of CA IX to specific antibodies and metabolon partners was investigated by competitive ELISA and proximity ligation assays, respectively.
\end{abstract}

Results: Carnosine increased the expression levels of HIF-1a and HIF targets and increased the extracellular pH, suggesting an inhibitory effect on CA IX-mediated acidosis. Moreover, carnosine significantly inhibited the growth of three-dimensional spheroids and tumor xenografts compared with untreated controls. Competitive ELISA showed that carnosine disrupted binding between CA IX and antibodies specific for its catalytic domain. This finding was supported by reduced formation of the functional metabolon of CA IX and anion exchanger 2 in the presence of carnosine.

Conclusions: Our results indicate that interaction of carnosine with CA IX leads to conformational changes of CA IX and impaired formation of its metabolon, which in turn disrupts CA IX function. These findings suggest that carnosine could be a promising anticancer drug through its ability to attenuate the activity of CA IX.

Keywords: Carbonic anhydrase IX, Hypoxia, Carnosine, pH regulation

\footnotetext{
* Correspondence: virupast@savba.sk

${ }^{1}$ Department of Molecular Medicine, Institute of Virology, Slovak Academy of

Sciences, Dubravska cesta 9, Bratislava 845 05, Slovak Republic

Full list of author information is available at the end of the article
} 


\section{Background}

Hypoxia in the tumor microenvironment is associated with poor prognosis and a poor response to therapy, underlying the importance of studying the effect of potential anticancer drugs on the hypoxia pathway. Stabilization of hypoxia-inducible factor 1 (HIF-1) as an adaptive response to hypoxic conditions in tissues results in transcriptional activation of many genes that play an important role in cancer-related processes, such as angiogenesis, cell survival, glucose metabolism, and cell invasion. HIF-1 is a heterodimer consisting of a constitutively expressed HIF$1 \beta$ subunit and a HIF- $1 \alpha$ subunit that is regulated through $\mathrm{O}_{2}$-dependent degradation modulated by prolyl hydroxylation. The von Hippel-Lindau (VHL) tumor-suppressor protein binds specifically to hydroxylated HIF- $1 \alpha$ which is then ubiquitylated by E3 ubiquitin-protein ligases and rapidly degraded by the proteasome [1]

The dipeptide $\beta$-alanyl-L-histidine, also known as carnosine, was described for the first time in the $19^{\text {th }}$ century [2]. Carnosine is naturally present in cardiac and skeletal muscles and the central nervous system, and is synthesized from $\beta$-alanine and L-histidine by carnosine synthase in muscle cells, glial cells, and oligodendrocytes [3]. Carnosine plays a role as a physiologic $\mathrm{pH}$ buffering substance and antioxidant [4]. It induces variable effects on the cardiovascular system, including down-regulation of blood pressure [5,6], inhibition of glycosylated lowdensity lipoprotein formation [7], and inhibition of angiotensin-converting enzyme activity [8]. It also acts as an anti-aging agent [9]. Moreover, it inhibits proliferation of cells derived from patients with glioblastoma [10] and the growth of tumors formed from neoplastic cell lines, such as Sarcoma-180 tumor cells [11], various neoplastic human and rodent cell lines [12], cells expressing the human epidermal growth factor receptor 2 (Her2/neu) [13], and HCT116 colon cancer cells [14]. Conversely, carnosine enhances the proliferation potential of cultured normal human fibroblasts, lengthens their lifespan, and suppresses senescence [9]. The mechanism of its action in tumor cells remains unclear.

Proteomic studies of glioblastoma cells after treatment with carnosine revealed significantly reduced expression of von Hippel-Lindau binding protein 1 (VBP1) [15], a protein that binds to the von Hippel-Lindau protein [16] and thus is linked to HIF-1 $\alpha$ signaling. Pretreatment with carnosine reduced the induction of HIF-1 $\alpha$ protein in $\mathrm{H} 9 \mathrm{c} 2$ cardiomyoblasts during hypoxia and further reduced its already low level under normoxia; the level of HIF-1 mRNA was transiently reduced after carnosine treatment, but increased after $24 \mathrm{~h}$ in a similar manner to controls. A similar experiment with human astrocytes showed that carnosine did not significantly alter the pattern of HIF- $1 \alpha$ protein expression in these cells [17].
Carbonic anhydrase IX (CA IX) is a membrane-bound metalloenzyme that is expressed in a broad range of solid tumors [18]. The main function of CA IX is to maintain intracellular $\mathrm{pH}$ homeostasis under hypoxic conditions that are common in solid tumors although it also modulates E-cadherin-mediated cell adhesion via its interaction with beta-catenin, which could be of potential significance in hypoxia-induced tumor progression [19]. CA IX contributes to ion transport and $\mathrm{pH}$ control by forming a bicarbonate transport metabolon with the sodium bicarbonate transporter $\mathrm{NBCe} 1$ and anion exchanger 2 (AE2) [20,21]. CA IX expression in tumors is recognized as a marker of hypoxia and an indicator of poor prognosis. Moreover, CA IX possesses clinical potential as a target for anticancer treatment [22]; indeed, functional inhibition of CA IX has been proposed as an attractive option for therapeutic targeting of various hypoxic tumors [18]. Transcription of the gene encoding CA IX is primarily activated by the hypoxia-inducible HIF-1 transcription factor that binds to the hypoxia response element (HRE) located next to the transcription initiation site [23]. Phosphorylation of Thr443 of CA IX by protein kinase A (PKA) in hypoxic cells is critical for its activation [21].

Because kinetic and X-ray crystallographic studies suggest that carnosine is a potent activator of the carbonic anhydrase isoforms hCA I, II, and IV [24] and the studies described above indicate that carnosine affects the HIF-1 signaling pathway, we initially examined whether CA IX is involved in the antitumor activity of carnosine. We subsequently investigated whether carnosine exerts its effect on CA IX through modulation of transcription and translation levels of HIF- $1 \alpha$ and CA IX and/or through altering CA IX function.

\section{Methods}

\section{Cell culture and spheroid preparation}

Madin-Darby canine kidney (MDCK), HeLa, HT-29, and $\mathrm{SiHa}$ cell lines were obtained from the American Type Culture Collection and cultured in Dulbecco's modified Eagle's medium (DMEM) supplemented with 10\% fetal calf serum (FCS; Bio Whittaker) and gentamicin (Sandoz) at $37^{\circ} \mathrm{C}$ and $5 \% \mathrm{CO}_{2}$ in humidified air. The cells were counted, seeded in 3- or 6-cm Petri dishes (Greiner) for $24 \mathrm{~h}$, and treated with L-carnosine (Sigma Aldrich) under normoxic (incubator, $\left.5 \% \mathrm{CO}_{2}\right)$ and hypoxic $\left(2 \% \mathrm{O}_{2}\right.$, $2 \% \mathrm{H}_{2}, 5 \% \mathrm{CO}_{2}, 91 \% \mathrm{~N}_{2}$, anaerobic workstation, Ruskinn Technology) conditions.

HeLa spheroids were generated by seeding cells $(1,250$ cells/well) in 96-well plates (Greiner) coated with $1 \%$ agarose. After 4 days of incubation at $37^{\circ} \mathrm{C}$ and $5 \% \mathrm{CO}_{2}$, the spheroids were photographed and treatment was initiated by addition of fresh medium with or without carnosine. In all experiments, at least 30 replicate wells 
were set up for the control and the carnosine treatment groups. Photographs were taken every $48 \mathrm{~h}$. At the end of the experiment, extracellular $\mathrm{pH}$ was measured and the spheroids were subjected to flow cytometric analysis to determine cell viability.

Measurement of extracellular $\mathrm{pH}$ using sensor dish reader The sensor dish reader (SDR; PreSense) monitors $\mathrm{pH}$ in real-time in special plates $\left(\right.$ HydroDish $\left.^{\circ}\right)$ using a noninvasive technique that detects the luminescence lifetime of a sensor spot at the bottom of each well that is dependent on the $\mathrm{pH}$ of the surrounding sample. Cells were seeded into wells and allowed to attach. Measurement was started on the second day, when the cells reached $80 \%$ confluence. Cells were cultured in the presence or absence of carnosine under hypoxic or normoxic conditions as described above. The $\mathrm{pH}$ was measured by the SDR every $30 \mathrm{~min}$.

\section{Competitive ELISA}

HeLa cells were cultured in 96-well plates for $24 \mathrm{~h}$ in normoxic conditions and then in hypoxic conditions for additional $24 \mathrm{~h}$, followed by 6 - $\mathrm{h}$ treatment with different concentrations of carnosine $(1.531,3.0625,6.125,12.25$, $25,50,100 \mathrm{mM}$ ) with or without specific antibodies against different domains of the CA IX protein (MAb10, MAb12) [25]. Cells were fixed with methanol, blocked with 10\% FCS in phosphate-buffered saline (PBS) for $30 \mathrm{~min}$, and incubated with HRP-conjugated secondary antibody for $1.5 \mathrm{~h}$ at room temperature. Absorbance and color changes were measured at $492 \mathrm{~nm}$.

\section{Immunofluorescence (IF)}

HeLa cells grown on glass coverslips were fixed in methanol. After blocking in 3\% bovine serum albumin (BSA)/PBS, the cells were incubated with primary antibodies against CA IX (M75 hybridoma medium) or against HIF- $1 \alpha$ (diluted in PBS with $0.5 \%$ BSA) for $1 \mathrm{~h}$ at $37^{\circ} \mathrm{C}$. The cells were washed four times for $10 \mathrm{~min}$ with PBS containing $0.02 \%$ Tween 20, incubated for $1 \mathrm{~h}$ at $37^{\circ} \mathrm{C}$ with Alexa-conjugated secondary antibody diluted in PBS with $0.5 \%$ BSA, and washed three times with PBS. All experiments were also performed in the absence of the primary, secondary, or both antibodies as negative controls. Nuclei were stained with 4',6-diamidino-2-phenylindole (DAPI; 1:36000; Sigma Aldrich) for $5 \mathrm{~min}$. Finally, the cells were mounted in Fluoroshield Mounting Medium (Abcam) and analyzed by laser scanning microscopy (LSM 510 Meta Microscope; Zeiss).

To investigate the influence of carnosine treatment on the binding of fluorescein isothiocyanate (FITC)-labeled CA specific inhibitor (FITC_CAI), HeLa cells were cultured without and with $20 \mathrm{mM}$ carnosine in normoxic and hypoxic conditions. After $48 \mathrm{~h}$, the medium was replaced by fresh medium containing FITC_CAI at a final concentration of $0.1 \mathrm{mmol} / \mathrm{L}$. After further incubation for $1 \mathrm{~h}$, the live cells were analyzed by laser scanning microscopy (LSM 510 Meta Microscope; Zeiss) using the incubation stage set at $37^{\circ} \mathrm{C}$ and $5 \% \mathrm{CO}_{2}$. FITC-labeled carbonic anhydrase specific inhibitor (FITC_CAI) [26] was a gift from Professor C.T. Supuran.

\section{Proximity ligation assay}

The proximity ligation assay (PLA) was used for in situ detection of the interaction between CA IX and AE2. The assay was performed in a humid chamber at $37^{\circ} \mathrm{C}$ according to the manufacturer's instructions (Olink Bioscience). $\mathrm{SiHa}$ cells were seeded on glass coverslips and allowed to attach before transfer to $2 \%$ hypoxia and further cultured for $24 \mathrm{~h}$. After starvation overnight in DMEM supplemented with $0.5 \%$ FCS, carnosine was added to selected samples (final concentration $20 \mathrm{mM}$ ) and the control and treated cells were cultured for an additional $24 \mathrm{~h}$ in hypoxia. The cells were fixed with methanol, blocked with $3 \%$ BSA/PBS for $30 \mathrm{~min}$, incubated with a mixture of antibodies against CA IX and AE2 for $1 \mathrm{~h}$, washed three times, and incubated with plus and minus PLA probes for $1 \mathrm{~h}$. The cells were washed, incubated with ligation mixture containing connector oligonucleotides for $30 \mathrm{~min}$, washed again, and incubated with amplification mixture containing fluorescently labeled DNA probe for $100 \mathrm{~min}$. After a final wash, the samples were mounted and the signal representing interaction between CA IX and AE2 was analyzed using a Zeiss LSM 510 Meta confocal microscope.

\section{Flow cytometry analysis (FACS)}

HeLa cells were treated with carnosine (5-40 mM) under normoxic and hypoxic conditions. After $48 \mathrm{~h}$, the cells were detached using trypsin, which was then inactivated by $10 \%$ FCS in PBS with 2 mM EDTA. Cells were centrifuged and resuspended in PBS with 10\% FCS at a final concentration of $1 \times 10^{6}$ cells $/ \mathrm{mL}$. For measurement of the surface expression of CA IX protein, $100 \mu \mathrm{L}$ of hybridoma medium containing a M75 antibody against CA IX was added to $100 \mu \mathrm{L}$ of the sample. After incubation at $4^{\circ}$ $\mathrm{C}$ for $30 \mathrm{~min}$, the cells were centrifuged, washed twice with PBS, and incubated with the secondary Alexa Fluor 488 donkey anti-mouse antibody. Cells stained with only secondary antibody were used as a negative control. For assessment of cell viability, the cells were stained with propidium iodide at a final concentration of $5 \mu \mathrm{g} / \mathrm{mL}$ and incubated for $5 \mathrm{~min}$ at room temperature. The samples were analyzed using a Guava EasyCyte Plus flow cytometer with Guava Express Pro 2.2.3 software (Millipore).

\section{Western blotting}

For western blotting (WB), cells grown in confluent monolayers were rinsed twice with cold PBS, resuspended 
in ice-cold lysis buffer (1\% Triton X-100; $50 \mathrm{mM}$ Tris $\mathrm{pH}$ 7,5; $150 \mathrm{mM} \mathrm{NaCl} ; 0,5 \%$ Nonidet P-40) containing protease (Roche) and phosphatase inhibitors cocktail (Sigma Aldrich), disrupted by sonication and cleared by centrifugation. Protein concentrations were quantified using the BCA protein assay reagents (Pierce). Protein extracts $(100 \mu \mathrm{g} / \mathrm{lane})$ were resolved in $8 \%$ SDS-PAGE and transferred to a polyvinylidene difluoride (PVDF) membrane (Macherey-Nagel). The total level of CA IX protein was detected by HRP-conjugated M75 antibody, and HIF- $1 \alpha$ and actin were detected using purified primary antibodies and the appropriate HRP-conjugated secondary antibodies as described in the section Antibodies. Protein bands were visualized using an enhanced chemiluminescence kit (GE Healthcare Bio-Sciences).

\section{Real-Time quantitative PCR (qPCR)}

HeLa cells were cultured with or without $20 \mathrm{mM}$ carnosine in normoxia and hypoxia for $48 \mathrm{~h}$. Total RNA was isolated using Instapure solution (Eurogentech) and reverse transcription of RNA was performed with the High-Capacity cDNA Reverse Transcription kit (Applied Biosystems) according to the manufacturer's recommendations. Amplification was performed in a Stratagene Mx 3005P thermal cycling block (Agilent Technologies). PCR was carried out in 20- $\mu \mathrm{L}$ volumes using Maxima Syber Green PCR Master Mix (Fermentas) for $10 \mathrm{~min}$ at $95^{\circ} \mathrm{C}$ for initial denaturation followed by 40 cycles of $95^{\circ} \mathrm{C}$ for $15 \mathrm{~s}$ and $60^{\circ} \mathrm{C}$ for $1 \mathrm{~min}$. Sample Ct values were normalized to actin. Relative expression was calculated using the $\Delta \Delta \mathrm{Ct}$ method. All amplifications were performed in triplicate in three independent experiments. Oligonucleotides used for real-time qPCR were as follows: HIF-1 $\alpha$ sense 5 '-GCTTGGTGCTG ATTTGTGAACC-3', HIF-1 $\alpha$ antisense 5'-GCATCCTG TACTGTCCTGTGGTG-3', Actin sense 5'-CCAACCGC GAGAAGATGACC-3', Actin antisense 5'-GATCTTCAT GAGGTAGTCAGT-3', GLUT-1 sense 5'-CTCCTTTCTC CAGCCAGCAATG-3', GLUT-1 antisense 5'-CCAGCAG AACGGGTGGCCATAG-3', VEGF sense 5'-CAGCACG GT CCCTCTTGGAA-3', VEGF antisense 5' -CCTCCTC TTCCCTGTCAGGA-3', VBP1 sense 5' -CTGTGGTTGG GGGCTAATGT-3', VBP1 antisense 5'-CCCTGGCCATA TTGACTTCTGT-3'.

\section{Chromatin immunoprecipitation (ChIP)}

HeLa cells were plated onto $10-\mathrm{cm}$ Petri dishes, cultured to approximately $70 \%$ monolayer density, and then incubated in the presence or absence of $20 \mathrm{mM}$ carnosine in hypoxic conditions for additional $48 \mathrm{~h}$. The cells were fixed in $1 \%$ formaldehyde directly in medium at room temperature $\left(\sim 21^{\circ} \mathrm{C}\right)$ for $15 \mathrm{~min}$. Chromatin isolation and immunoprecipitation with antibody against HIF- $1 \alpha$ were performed using Exacta ChIP (R\&D Systems) according to the manufacturer's instructions. DNA was purified using the Wizard SV Gel and PCR Clean-Up System (Promega). Amplification of the samples was performed with HF Phusion polymerase (Thermo Fisher) in an automatic DNA thermal cycler (Eppendorf) using initial denaturation at $98^{\circ} \mathrm{C}$ for $9 \mathrm{~min}$ followed by 43 cycles of denaturation at $97^{\circ} \mathrm{C}$ for $1 \mathrm{~min}$ and annealing at $62^{\circ} \mathrm{C}$ for 1 min. Primers flanking the HRE elements within the $C A 9$ and VEGF promoter were as follows: $h C A 9$ HRE sense 5'TCCTAGCTTTGGTATGGGGGAGAG-3', hCA9 HRE antisense 5'-AGTGACAGCAGCAGTTGCACAGT-3', hVEGF HRE sense $5^{\prime}$-CCTCAGTTCCCTGGCAACAT CTG-3', hVEGF HRE antisense 5'-CCTCAGTTCCCT GGCAACATCTG-3'.

\section{Animal experiments}

CD1 nude mice were purchased from Charles River Laboratories. The animals had access to standard food and water ad libitum. Ten male animals were injected subcutaneously into the flank on both sides with $2 \times 10^{6}$ HeLa cells in $100 \mu \mathrm{L}$ sterile PBS. At 14 days after implantation, the animals were divided into two groups: the first group (five animals) was treated with carnosine (50 $\mu \mathrm{L}$ of $1 \mathrm{M}$ stock, dissolved in sterile PBS) administered subcutaneously $2 \mathrm{~cm}$ from the implantation site every second day, and the second group (five animals) was used as a control (sterile PBS only). Tumor size was determined by caliper measurements and was calculated according to the formula $\mathrm{W}^{2}{ }^{*} \mathrm{~L} / 2$, where $\mathrm{W}$ is the width and $L$ the length of the tumor. All animal protocols were approved by the Institutional Ethics Committee of the Institute of Virology and the State Veterinary and Food Institute of the Slovak Republic (Permit no. 4245/13-221).

\section{Immunohistochemistry (IHC)}

Tumor specimens were fixed in formalin, dehydrated in an ethanol series, treated with xylene, and mounted in paraffin. Serial sections of tissues were cut and deparaffinized in a xylene and ethanol series. Immunostaining for HIF- $1 \alpha$ was performed after antigen retrieval $\left(125^{\circ} \mathrm{C}\right.$ for $5 \mathrm{~min}, 95^{\circ} \mathrm{C}$ for $10 \mathrm{~min}$ in citrate buffer, $\mathrm{pH}$ 6) using the Dako Cytomation Catalyzed Signal Amplification System kit (Dako). CA IX staining was performed using Dako EnVision + System-HRP (Dako). Cell nuclei were counterstained with hematoxylin solution.

\section{Antibodies}

Primary antibodies: mouse monoclonal anti-human HIF$1 \alpha$ (dilutions used: WB 1:250, IF 1:150, IHC 1:150; BD Transduction Laboratories); goat polyclonal anti-human actin (WB 1:1000; Santa Cruz Biotechnology); rabbit antihuman AE2 (PLA 1:500) [20]; mouse monoclonal antihuman carbonic anhydrase IX-M75 hybridoma medium (PLA, FACS, IF non-diluted; IHC 1:100); purified mouse monoclonal anti-human carbonic anhydrase IX-MAb10, 
MAb12 (ELISA $200 \mu \mathrm{g} / \mathrm{mL}$ ) [25]; purified M75 antibody against CA IX conjugated with HRP (WB 1:7500).

Secondary antibodies: Alexa Fluor 488-conjugated donkey anti-mouse IgG (IF 1:1000, FACS 1:3000; Invitrogen); HRP-conjugated goat anti-mouse IgG (WB 1:5000; Dako); HRP-conjugated rabbit anti-goat IgG (WB 1:5000; Dako).

\section{Results}

\section{Carnosine reduces CAIX-mediated acidification}

Cultivation of HeLa cells under hypoxia for $48 \mathrm{~h}$ in the presence of carnosine $(5-40 \mathrm{mM})$ resulted in reduced acidification of the extracellular environment in a dosedependent manner (Figure 1A). The effect of carnosine on HeLa cells in normoxic conditions was substantially smaller (data not shown). Because of its physiologic relevance, a carnosine concentration of $20 \mathrm{mM}$ was selected for further tests on different cancer cell lines (SiHa, HeLa, HT-29). Incubation with carnosine markedly reduced the acidification of growth media in hypoxic conditions for all cell lines studied (Figure 1B, Additional file 1). We also observed a carnosine-mediated decrease in acidification in MDCK cells transfected with CA IX, whereas the effect of carnosine on their mock-transfected counterparts was considerably smaller.

\section{Effect of carnosine on the level of total CA IX}

To determine whether the carnosine-mediated reduction in extracellular acidification of CA IX-positive cells is related to CA IX protein level, we cultivated HeLa cells in hypoxic conditions and used our in-house anti-CA IX antibody M75 to measure CA IX protein levels. The level of CA IX protein increased after carnosine treatment (Figure 2A). This result was confirmed by immunofluorescent staining of CA IX (Figure 2B) and by flow cytometry analysis, which showed that $20 \mathrm{mM}$ carnosine treatment increased the levels of surface CA IX in HeLa cells under hypoxia (Figure 2C). Carnosine did not change the degree of phosphorylation at Thr443, suggesting that

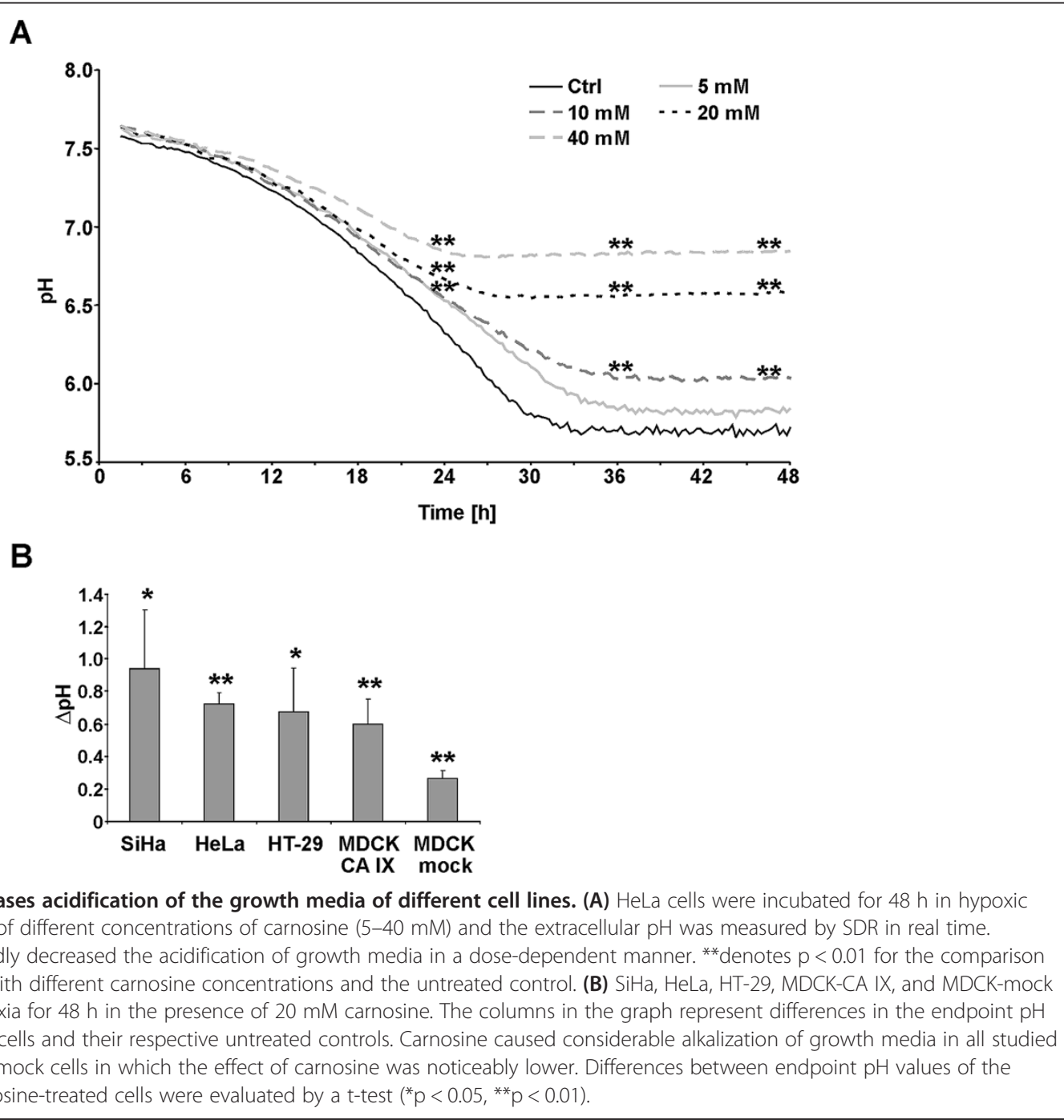




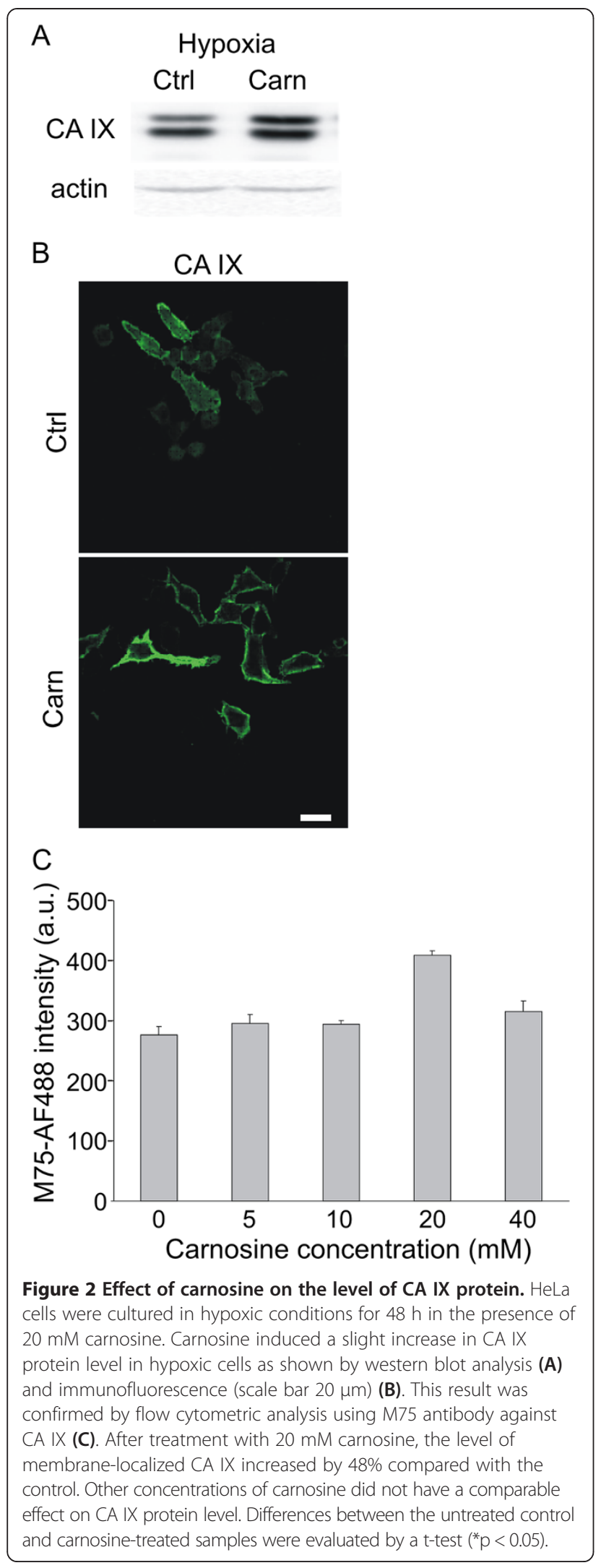

it has no effect on activation of CA IX through phosphorylation by PKA (Additional file 2).

\section{Carnosine treatment increases the level of HIF-1a protein} and mRNA and the expression of hypoxia-regulated genes Because transcription of CA IX is activated by HIF- $1 \alpha$, we tested whether carnosine influenced HIF-1 $\alpha$ protein and mRNA levels in HeLa cells. HeLa cells were cultured in hypoxic conditions for $48 \mathrm{~h}$ with or without $20 \mathrm{mM}$ carnosine. Western blot analysis showed a significant increase in HIF- $1 \alpha$ signal in cells treated with carnosine compared with controls (Figure 3A). This finding was supported by immunofluorescent staining of HIF- $1 \alpha$, which showed a stronger HIF-1 $\alpha$ signal in the nuclei of treated cells (Figure 3B). Data from qPCR analysis confirmed an increased level of HIF-1 $\alpha$ mRNA after carnosine treatment under hypoxia compared with the untreated control (Figure 3C). The activity of HIF- $1 \alpha$ was demonstrated by the increase in mRNA expression of the HIF- $1 \alpha$ targets vascular endothelial growth factor (VEGF) and glucose transporter 1 (GLUT-1) after carnosine treatment in hypoxia (Figure 3C). Moreover, ChIP analysis showed a moderate increase in binding of HIF- $1 \alpha$ to the HRE in both $C A 9$ and $V E G F$ promoters (Figure 3D). Interestingly, the level of $V B P 1$ mRNA decreased after carnosine treatment compared with the control (Figure 3C), indicating reduced degradation and increased stabilization of HIF- $1 \alpha$ protein.

Carnosine inhibits binding of CA-specific inhibitor and CA IX-specific antibodies and impairs formation of the CA IX metabolon

We next investigated binding of fluorescein-conjugated CA-specific homosulfanilamide inhibitor (FITC_CAI) to carnosine-treated and untreated cells in hypoxic conditions. Svastova et al. previously showed that FITC_CAI binds only to hypoxic cells expressing CA IX, and it is widely accepted that this inhibitor binds only to catalytically active CA IX that has been activated by hypoxia [26]. We observed a reduction in the immunofluorescent signal of FITC-CAI after carnosine treatment of HeLa cells (Figure 4A) and MDCK-CA IX cells (data not shown) under hypoxia, indicating a decrease in CA IX activity in the presence of carnosine. This assumption is supported by the results of competitive inhibition ELISA performed in HeLa cells after culture in the presence of different concentrations of carnosine together with the CA IX-specific antibodies MAb10 and MAb12 directed against conformational epitopes in the catalytic domain of CA IX. As shown in Figure 4B, carnosine inhibited the binding of MAb10 and MAb12. Furthermore, a proximity ligation assay showed that carnosine treatment reduced the signal arising from direct interaction of $\mathrm{CA}$ IX and AE2 in the metabolon of SiHa cells (Figure 4C). 


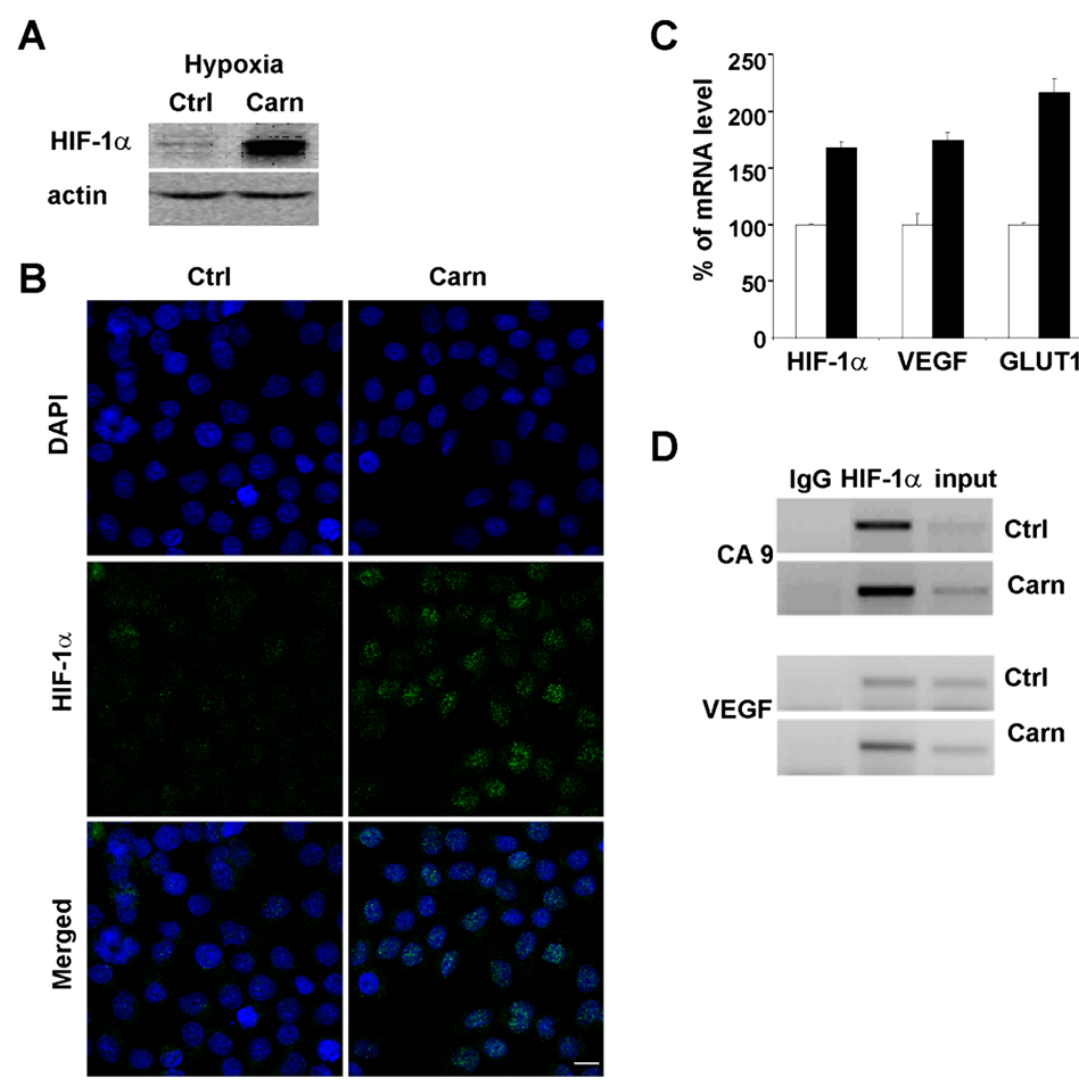

Figure 3 Effect of carnosine on the level of HIF-1a. HIF-1a protein level was increased in hypoxic HeLa cells cultured in the presence of $20 \mathrm{mM}$ carnosine for $48 \mathrm{~h}$ as demonstrated by western blot (A) and immunofluorescence (scale bar, $10 \mu \mathrm{m})$ (B). (C) Carnosine treatment increased expression of HIF-1 1 mRNA and its target genes VEGF and GLUT-1 under hypoxic conditions. Concurrently, the mRNA level of VBP1, a protein that binds to VHL and is involved in HIF-1a degradation, was decreased. (D) Chromatin immunoprecipitation assay performed under the same conditions demonstrated that HIF-1a bound to hypoxia-responsive elements in the promoter region of VEGF and CA9 genes.

\section{Carnosine treatment reduces spheroid size and cell viability}

To confirm the effect of carnosine in a physiologically more relevant three-dimensional (3D) environment, we treated spheroids formed by HeLa cells with carnosine added to the culture medium only after the spheroids had already formed, or with carnosine present during the period of spheroid formation. Both experimental groups formed spheroids, indicating that spheroid formation was not significantly affected by carnosine. At the end of the experiment the carnosine-treated spheroids in both groups had a significantly smaller (almost $50 \%$ smaller) diameter than the controls; moreover, the extracellular $\mathrm{pH}$ of the treated groups was higher in the treated cultures than in the controls (Figure 5A, 5B).

Data from flow cytometric analysis showed that carnosine treatment of a two-dimensional monolayer culture decreased the viability of hypoxic cells in a dose-dependent manner: $5 \mathrm{mM}$ carnosine decreased HeLa cells viability only slightly, $10 \mathrm{mM}$ carnosine by approximately $10 \%$, and $20 \mathrm{mM}$ by approximately $15 \%$ (Figure $5 \mathrm{C}$ ). In comparison, the viability of HeLa cells in normoxic conditions remained relatively constant in the presence of different concentrations of carnosine (data not shown). In 3D culture, where hypoxia develops in the center of spheroids, we observed a marked decrease in viability of HeLa spheroids of $50 \%$ after treatment with $20 \mathrm{mM}$ carnosine compared with the controls (Figure 5D).

\section{Carnosine reduces tumor size in an experimental mouse xenograft model}

Tumor growth was visible 7 days after subcutaneous implantation of HeLa cells in all animals. On the $14^{\text {th }}$ day of the experiment, we separated the mice into two groups and started subcutaneous administration of carnosine solution to animals in the carnosine group. At the same time, we commenced caliper measurement of the tumors. All animals had comparable-sized tumors at the start of carnosine treatment. Between the $21^{\text {st }}$ and $28^{\text {th }}$ day of the experiment we noticed faster growth of tumors in the control group compared with the carnosine-treated group, in which the average tumor size remained relatively constant. Although several tumors continued to grow in the carnosine-treated group, the rate of tumor growth was 


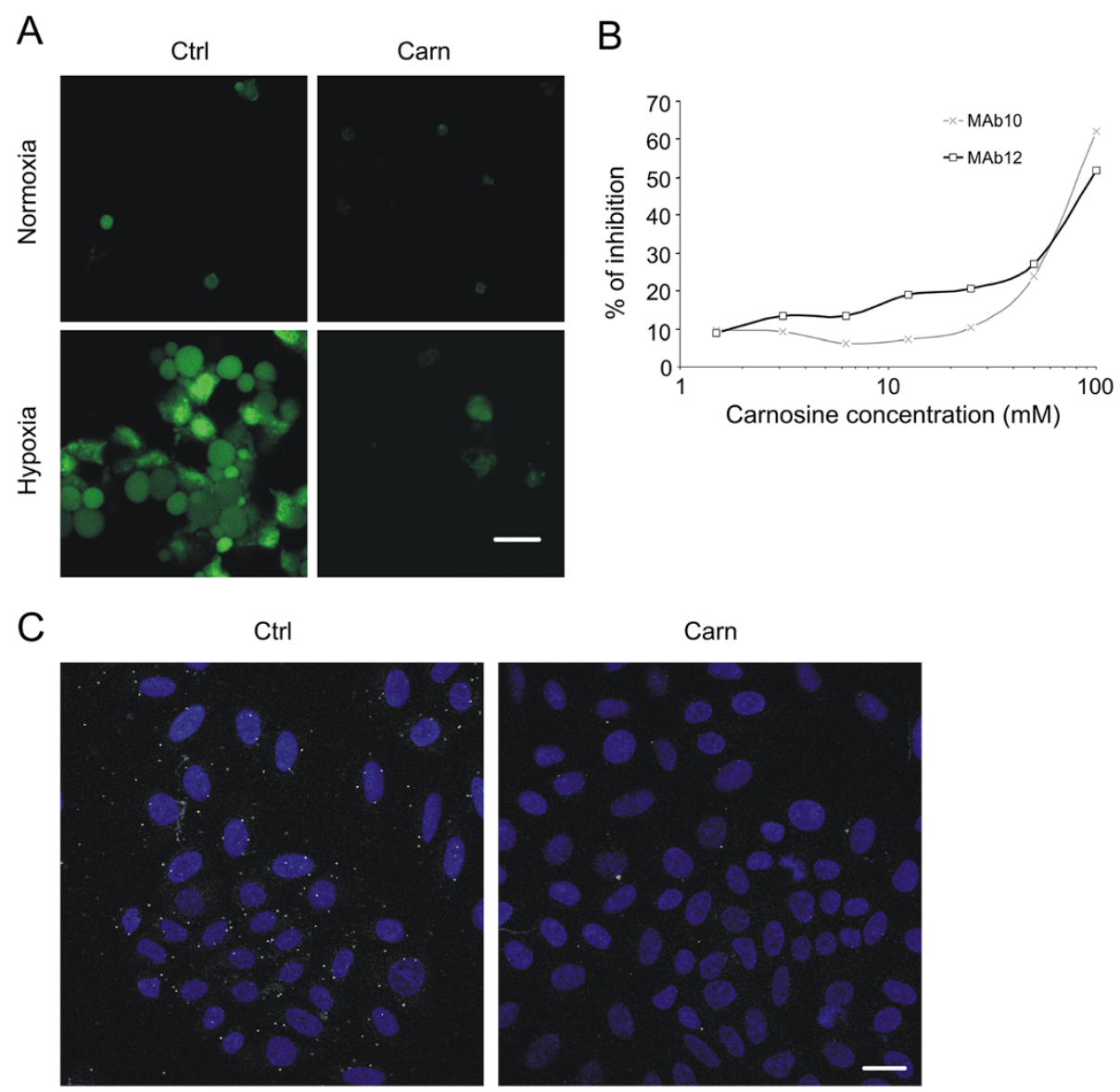

Figure 4 Carnosine impairs binding of CA IX-specific inhibitor and antibodies and affects formation of a CA IX metabolon.

(A) Immunofluorescence signal of FITC-labeled CA specific inhibitor, which binds only to hypoxic cells with catalytically active CA IX, was markedly reduced after treatment of HeLa cells with $20 \mathrm{mM}$ carnosine in hypoxia for $48 \mathrm{~h}$. (B) Graph of competitive inhibition ELISA showing the percentage of inhibition after cultivation of HeLa cells with different concentrations of carnosine together with specific CA IX antibodies MAb10 and MAb12. Binding of antibodies to conformational epitopes in the catalytic domain of CA IX was inhibited by carnosine in a concentration-dependent manner. (C) SiHa cells cultured in islands under hypoxic conditions were analyzed by proximity ligation assay to visualize direct interaction between CA IX and the anion exchanger AE2 in the functional metabolon. The positive signal represented by bright white dots was considerably reduced in cells treated with $20 \mathrm{mM}$ carnosine for $24 \mathrm{~h}$, supporting the proposal that binding of carnosine alters the conformation of CA IX. Scale bar, $20 \mu \mathrm{m}$.

very slow, whereas the growth of some tumors stopped and several tumors even became smaller (Figure 6A). These observations were confirmed after the final examination, when we found a significant difference in the weight of tumors between the control and carnosinetreated groups (Figure 6B). Immunohistochemical staining of formalin-fixed, paraffin-embedded tumor tissues demonstrated enhanced intensity of HIF1- $\alpha$ and CA IX staining in the carnosine-treated group (Figure $6 \mathrm{C}$ ). These results correspond with the higher level of both proteins observed in vitro.

\section{Discussion}

Identification of a potent anticancer drug that does not have adverse side effects remains a highly topical issue. The clinical use of carnosine in children with autism [27] and the use of a zinc complex of L-carnosine (Polaprezinc) as an anti-ulcer drug in Japan since 1994 have yielded encouraging results [28]. Application of this dipeptide in anti-cancer therapy is still an emerging field, but carnosine seems to be a promising candidate because of its antitumorigenic effects combined with its natural presence in the body and its beneficial influence on normal cells. These positive effects include protection of astroglial cells by NO trapping [29], protection against hypoxia-ischemia brain damage [30], reduction in the development of inflammation and tissue injury associated with spinal cord trauma [31], protection of lung tissue against bleomycininduced injury [32], prevention of vascular damage in experimental diabetic retinopathy [33], and protection against ionizing radiation [34,35].

There is a lack of published data on carnosine administration in human participants in the setting of cancer treatment. Two recently published articles report no 


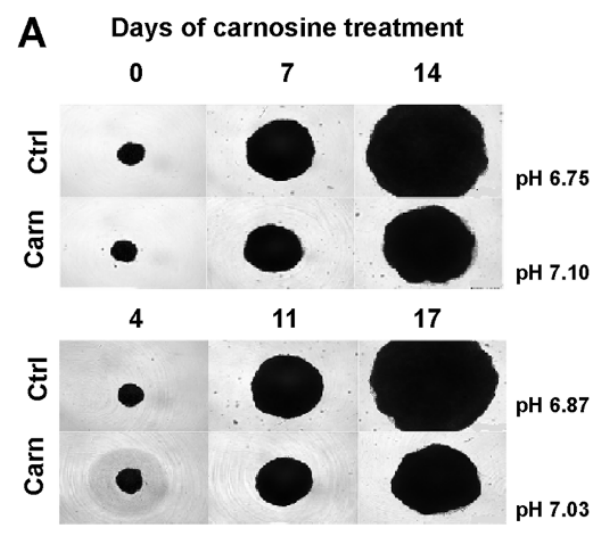

C

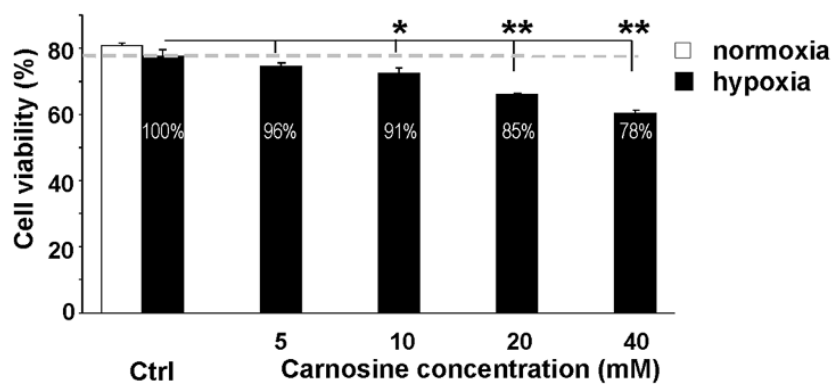

B

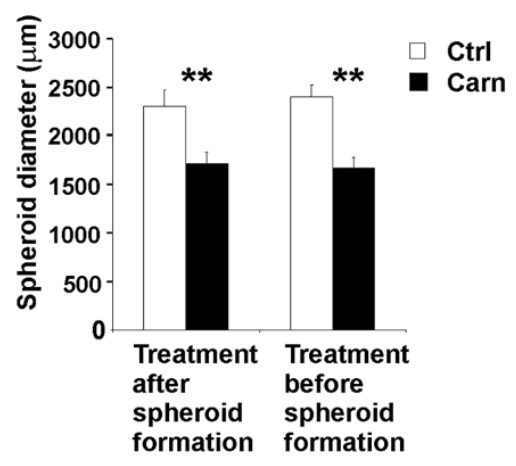

D

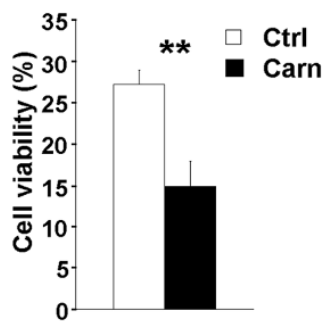

Figure 5 Effect of carnosine on spheroid size and cell viability. (A) Images of spheroids cultured in the presence and absence of $20 \mathrm{mM}$ carnosine. Carnosine was added after spheroids had already formed (upper image) or before spheroid formation (lower image). The images show the same time points of spheroid growth, although the duration of carnosine treatment differs as indicated, depending whether carnosine was added before or after spheroid formation. $\mathrm{pH}$ values represent measured $\mathrm{pH}$ of media collected from all respective spheroid samples ( $\mathrm{N}>30$ ) at the end of the experiment. (B) In both experimental conditions, the diameters of carnosine-treated spheroids were significantly smaller than those of controls (t-test, $\left.{ }^{* *} p<0.01\right)$. (C) Flow cytometry showed that treatment with different concentrations of carnosine $(5-40 \mathrm{mM})$ decreased cell viability in hypoxic conditions in a dose-dependent manner (t-test, ${ }^{*} \mathrm{p}<0.05,{ }^{* *} \mathrm{p}<0.01$ ). Numbers in the columns give the ratio for the viability of a carnosine-treated sample and the respective hypoxic control (set as 100\%). The ratio of the viability of hypoxic and normoxic controls was $96 \%$. (D) Cultivation of HeLa spheroids in the presence of $20 \mathrm{mM}$ carnosine significantly reduced cell viability by approximately $50 \%$ compared with the control group (t-test, $\left.{ }^{* *} \mathrm{p}<0.01\right)$.

adverse effects for carnosine dosages of $4 \mathrm{~g}$ as a single dose [36] or $400 \mathrm{mg}$ twice daily [27]. These data indicate the feasibility of carnosine as a therapeutic agent, although more clinical trials are clearly needed. In this article, we focused on the effect of carnosine on the hypoxic pathway, which is often up-regulated in tumors, and specifically on its effect on CA IX, expression of which is often associated with solid hypoxic tumors. The major function of $\mathrm{CA}$ IX in cancer is maintenance of $\mathrm{pH}$ homeostasis, which is related to the acidification of the tumor microenvironment that promotes cancer cell migration and invasiveness. Our results showed that carnosine causes a shift in extracellular $\mathrm{pH}(\mathrm{pHe})$ from acidic to alkaline for various cancer cell lines (HeLa, SiHa, HT-29) under hypoxic conditions. Although carnosine functions as a physiologic buffer, the observed change in pHe cannot be attributed to its buffering capacity alone. $\mathrm{pH}$ measurements of mock-transfected and CA IX-transfected MDCK cells demonstrated that carnosine treatment leads to a reduction of CA IX acidification activity, indicating a direct influence of carnosine on CA IX function.

Transcription of CA9 is induced by the binding of HIF- $1 \alpha$ to its core promoter [23]. Available information on the effect of carnosine on HIF-1 $\alpha$ is very limited. Using a gene reporter assay, Oppermann et al. found enhanced activity of HIF-1 $1 \alpha$ in the presence of carnosine and hypoxic conditions in the glioblastoma cell lines T98G, LN405, and 1321N1 and in one primary culture [37]. In contrast, Bharadwaj et al. showed that carnosine treatment decreased HIF-1 $\alpha$ expression in H9c2 cardiomyoblasts, but not in human astrocytes; however, neither of these cell lines is cancerous [17]. In our study, carnosine treatment increased protein and mRNA levels of HIF- $1 \alpha$, and its effect on HIF- $1 \alpha$ activity was demonstrated by up-regulation of the target genes CA9, VEGF, and GLUT1 through the HRE element in their promoters. Degradation of HIF- $1 \alpha$ 


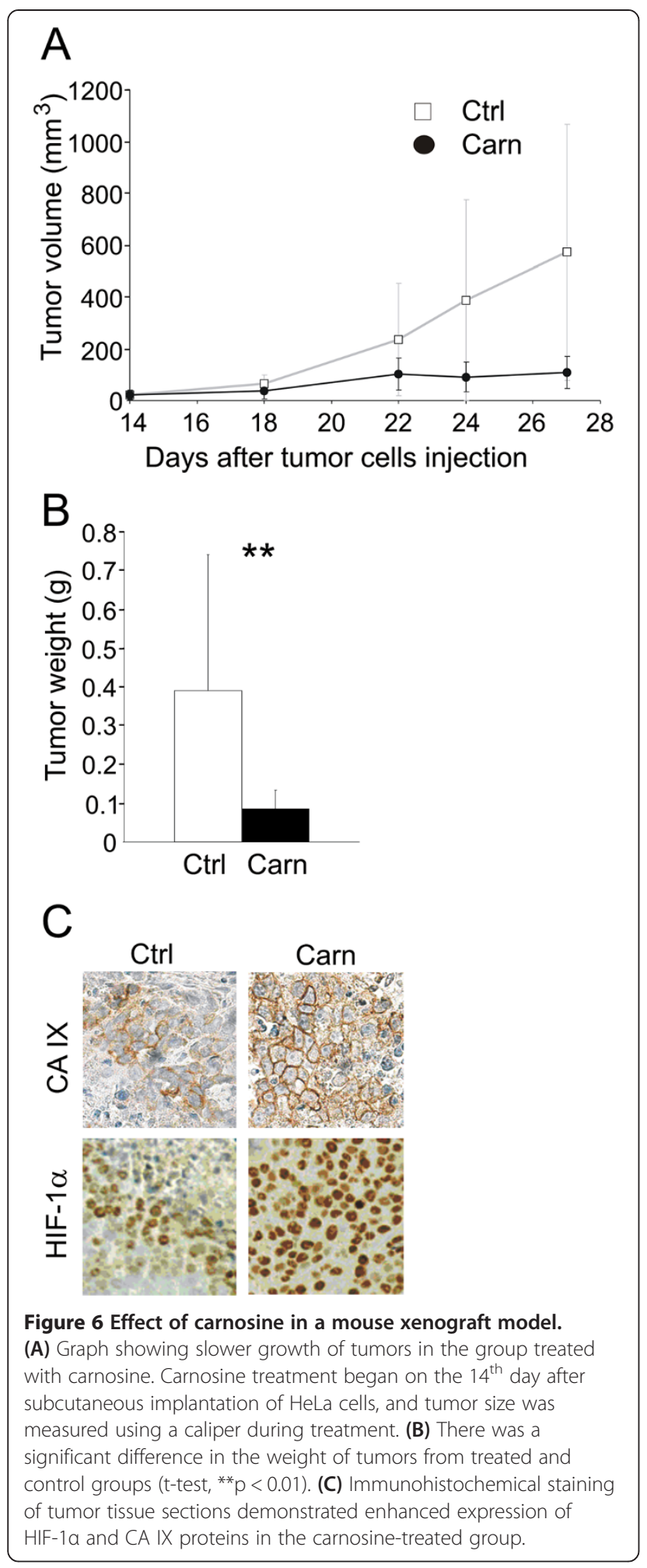

protein is mediated by the VHL tumor-suppressor protein, which interacts with von Hippel-Lindau binding protein 1 (VBP1) and forms a complex that is transported to the nucleus or cytoplasm. Proteomic studies of glioblastoma cells treated with carnosine showed significantly reduced expression of VBP1 protein and mRNA [15]. This is consistent with our findings that the level of VBP1 mRNA decreased in hypoxic HeLa cell monolayers after treatment with carnosine. Hence, the increase in HIF-1 $\alpha$ protein level after carnosine treatment could be a consequence of its stabilization due to reduced VHL-mediated degradation.

Hypoxia plays an important role in cancer progression and metastasis [38,39] and there is growing evidence that altered tumor metabolism and HIF-1-regulated enzymes such as CA IX and CA XII may be vital in the process of primary tumor progression to metastasis. Tumor-specific expression of CA IX and its association with tumor invasiveness and poor treatment outcome has led to interest in targeting this enzyme for cancer therapy $[22,40]$. CA IX activity plays an important role in the survival of tumor cells in hypoxic regions of solid tumors through the neutralization of intracellular $\mathrm{pH}$ and consequential acidification of the extracellular environment [26]. Because increased expression of HIF- $1 \alpha$ and CA IX in various tumors is believed to be associated with poor prognosis of cancer patients, an understanding of their involvement in the induction of tumor cell proliferation and consequent tumor growth has clinical relevance.

Carnosine inhibits the growth of tumors formed from different neoplastic cell lines [11-14]. Consistent with these findings, we observed an approximately 50\% decrease in the size of carnosine-treated HeLa spheroids and reduced growth of tumors in carnosine-treated animals. Our data from immunoblotting, flow cytometry, and immunofluorescence analyses showed a slight increase in CA IX protein level after carnosine treatment in accordance with the increase in HIF- $1 \alpha$, suggesting that the anti-tumor effect of carnosine cannot be explained by modulation of the amount of CA IX. Flow cytometric analysis showed that carnosine markedly reduced the viability of cells in hypoxic monolayers as well as in a 3D cellular model in which hypoxia naturally develops. The impaired viability of carnosine-treated cells is at least partially attributable to the effect of carnosine on CA IX catalytic activity and its implications for extracellular $\mathrm{pH}$.

In conditions of hypoxia, and the consequent acidosis linked to high production of lactic acid, accurate regulation of intracellular $\mathrm{pH}(\mathrm{pHi})$ could represent a key process that allows a cell to escape damage induced by these unfavorable conditions. Importantly, a change in $\mathrm{pHi}$ of approximately 0.1 units could induce specific effects in several processes crucial for cell homeostasis, such as ATP production, cell proliferation, and protein synthesis.

Adaptation of tumor cells to hypoxia is a complex process involving many metabolic and regulatory pathways. The different effect of carnosine on normal and tumor cells might be associated with metabolic differences between these cells. Normal cells derive the maximum possible energy from glucose by oxidizing it completely to 
$\mathrm{CO}_{2}$ (32 moles ATP) and if an adequate oxygen supply is not available they use anaerobic glycolysis to form lactate as the end product ( 2 moles ATP). In contrast, tumor cells preferentially use the anaerobic pathway. It is possible that carnosine inhibits glycolytic metabolism prior to the formation of triose phosphate by stimulating the activity of fructose 1,6-biphosphate, thus creating a fruitless ATPconsuming cycle [13,41]. In support of this, the amount of ATP in a HeLa cell monolayer was markedly reduced after carnosine treatment in hypoxia (data not shown), and a similar mode of action was observed in studies on different cancer cell lines [14,42]. This depletion of ATP could result in reduced cell proliferation.

The fact that carnosine treatment resulted in a decreased ability of CA IX-expressing cells to acidify their extracellular environment indicates that carnosine affects CA IX catalytic function. This is supported by reduced binding of the homosulfanilamide CA inhibitor (CAI) to carnosinetreated cells. Fluorescein-conjugated CAI could bind only to hypoxic cells that expressed CA IX, evoking the idea that hypoxia induces catalytic activity of CA IX by modulating CA IX folding in a manner that opens the active site and makes it accessible to the inhibitor [26]. Other studies showing that binding of the inhibitor was markedly reduced after reoxygenation of cells also indicate that sulfonamide-based inhibitors accumulate on CA IX-positive cells only under hypoxic conditions [43]. Our results further support an interaction between carnosine and CA IX protein. The specific antibodies MAb10 and MAb12 used in this study react with conformational epitopes in the catalytic domain [25]. The results of competitive ELISA indicated that direct binding of carnosine to CA IX influenced CA IX conformation and reduced binding of these specific antibodies. A change in CA IX conformation might also affect interactions of CA IX with its protein partners in a metabolon and thus modulate CA IX activity. Indeed, impaired formation of a bicarbonate transport metabolon was demonstrated by a reduced signal in the proximity ligation assay between CA IX and AE2.

Although the application of carnosine in clinical settings, especially as an anti-neoplastic therapeutic, has been discussed for several years, experimental-based explanations of its effects are still insufficient and no double-blind clinical trials have been performed [44]. Nagai and Suda first described the anti-neoplastic effects of carnosine on Sarcoma-180 cells implanted subcutaneously into ddY mice [45]. Renner et al. showed that carnosine delays aggressive tumor growth in nude mice after subcutaneous implantation of cells expressing human epidermal growth factor receptor 2 by affecting proliferation in vivo [13]. They also demonstrated that carnosine inhibits growth of cells from human malignant glioma and identified carnosine as an inhibitor of anaerobic glycolysis that is vital for the growth of gliomas [42]. A recent study

revealed that carnosine inhibited tumor proliferation of human colon cancer cells transplanted into athymic nude mice, probably by elevating natural killer activity of splenic cells [46]. Carnosine was also shown to inhibit KRASmediated HCT116 proliferation [14], to inhibit metastasis of SK-Hep-1 invasive hepatocarcinoma cells by inhibiting expression and activity of matrix metalloproteinase 9 [47], and to eliminate tumor cells from a mixture of normal fibroblasts and HeLa cells [12].

As CA IX expression is predominantly associated with tumors and is often a marker of poor prognosis, the possible inhibition of its activity by carnosine deserves further investigation because it could lead to anticancer activity without blocking the expression of HIF-1 $\alpha$. The fact that the binding of monoclonal antibodies to CA IX is not inhibited at physiologic concentrations of carnosine can be perceived as an advantage as it enables the use of immunotherapy with parallel blocking of CA IX function by carnosine. The induction of CA IX protein expression by carnosine could also be a positive side effect, as it creates better conditions for immunotherapy through increasing the number of target molecules.

\section{Conclusions}

CA IX is often expressed in solid tumors and is considered a marker of hypoxia and an indicator of poor prognosis. Carnosine reduces the extracellular acidosis linked to catalytic activity of CA IX in hypoxia and inhibits the growth of spheroids and tumor xenografts. Our results suggest that the interaction of carnosine with CA IX leads to conformational changes of the CA IX protein and impairs formation of its metabolon, which in turn influences its function. Thus, carnosine could be a promising anticancer drug through its ability to attenuate the activity of CA IX.

\section{Additional files}

\section{Additional file 1: SiHa, HeLa, HT-29, MDCK CA IX and MDCK mock cells were exposed to hypoxia for $\mathbf{4 8}$ hours in the presence of $\mathbf{2 0}$ $\mathrm{mM}$ carnosine. The columns in the graph represent endpoint values of $\mathrm{pH}$ of carnosine treated cells and their respective untreated controls. Carnosine caused a considerable alkalization of growth media in all studied cell lines, except for MDCK mock cells where the effect of carnosine was noticeably lower. Differences between control and carnosine treated cells were evaluated by t-test $\left({ }^{*} p<0.05,{ }^{* *} p<0.01\right)$.}

Additional file 2: Western blot representing the expression level of total CA IX protein and its phosphorylated form at threonine 443 in the intracellular tail in HeLa cells cultured in hypoxia with and without $20 \mathrm{mM}$ carnosine. Numbers underneath the protein bands give the increase in the signal after carnosine treatment compared to untreated cells. Carnosine did not cause increased phosphorylation of T443 as the increase of levels of PT433 and total CA IX is comparable.

\section{Competing interests}

The authors declare that this research was conducted in the absence of any commercial or financial relationship that could be construed as a potential conflict of interest. 


\section{Authors' contributions}

ZD and JP conceived and designed the experiments; ZD, PD, ML, FI, VS, MZ, and LC performed the experimental work and participated in data analysis; $Z D, L C$, and VS drafted the manuscript; JP and SP critically revised the manuscript and made many conceptual suggestions. All authors read and approved the final manuscript.

\section{Acknowledgments}

This work was supported by the following grants: APW-0893-11, VEGA 2/0129/11, VEGA 2/0134/12, and 7th Framework program of EU (Collaborative project METOXIA).

\section{Author details}

'Department of Molecular Medicine, Institute of Virology, Slovak Academy of Sciences, Dubravska cesta 9, Bratislava 845 05, Slovak Republic. ${ }^{2}$ Center for Molecular Medicine, Slovak Academy of Sciences, Vlarska 3-7, Bratislava 831 01, Slovak Republic.

Received: 19 December 2013 Accepted: 12 May 2014

Published: 22 May 2014

\section{References}

1. Semenza GL: Targeting HIF-1 for cancer therapy. Nat Rev Cancer 2003, 3(10):721-732

2. Gulewitsch W, Amiradzibi S: Ueber das Carnosin, eine neue organische Base des Fleischeextraktes. Ber Deut Chem Ges 1900, 33:1902-1903.

3. Bauer K: Carnosine and homocarnosine, the forgotten, enigmatic peptides of the brain. Neurochem Res 2005, 30(10):1339-1345.

4. Quinn PJ, Boldyrev AA, Formazuyk VE: Carnosine - its properties, functions and potential therapeutic applications. Mol Aspects Med 1992, 13(5):379-444.

5. Nagai K, Niijima A, Yamano T, Otani H, Okumra N, Tsuruoka N, Nakai M, Kiso Y: Possible role of L-carnosine in the regulation of blood glucose through controlling autonomic nerves. Exp Biol Med 2003, 228(10):1138-1145.

6. Tanida M, Niijima A, Fukuda Y, Sawai H, Tsuruoka N, Shen J, Yamada S, Kiso Y, Nagai K: Dose-dependent effects of $L$-carnosine on the renal sympathetic nerve and blood pressure in urethane-anesthetized rats. Am J Physiol Regul Integr Comp Physiol 2005, 288(2):447-455.

7. Rashid I, Van Reyk DM, Davies MJ: Carnosine and its constituents inhibit glycation of low-density lipoproteins that promotes foam cell formation in vitro. FEBS Lett 2007, 581(5):1067-1070.

8. Nakagawa K, Ueno A, Nishikawa Y: Interactions between carnosine and captopril on free radical scavenging activity and angiotensin-converting enzyme activity in vitro. Yakugaku Zasshi 2006, 126(1):37-42.

9. Hipkiss AR: On the enigma of carnosine's anti-ageing actions. Exp Gerontol 2009, 44(4):237-242.

10. Renner C, Seyffarth A, De Arriba SG, Meixensberger J, Gebhardt R, Gaunitz F: Carnosine inhibits growth of cells isolated from human glioblastoma multiforme. Int J Pept Res Ther 2008, 14(2):127-135.

11. Nagai K, Suda T: Antineoplastic effects of carnosine and beta-alanine -physiological considerations of its antineoplastic effects. Nihon Seirigaku Zasshi 1986, 48(11):741-747.

12. Holliday R, McFarland GA: Inhibition of the growth of transformed and neoplastic cells by the dipeptide carnosine. Br J Cancer 1996, 73(8):966-971.

13. Renner C, Zemitzsch N, Fuchs B, Geiger KD, Hermes M, Hengstler J, Gebhardt R, Meixensberger J, Gaunitz F: Carnosine retards tumor growth in vivo in an NIH3T3-HER2/neu mouse model. Mol Cancer 2010, 9:2.

14. Iovine B, lannella ML, Nocella F, Pricolo MR, Bevilacqua MA: Carnosine inhibits KRAS-mediated HCT116 proliferation by affecting ATP and ROS production. Cancer Lett 2012, 315(2):122-128.

15. Asperger A, Renner C, Menzel M, Gebhardt R, Meixensberger J, Gaunitz F: Identification of factors involved in the anti-tumor activity of Carnosine on glioblastomas using a proteomics approach. Cancer Invest 2011, 29(4):272-281

16. Tsuchiya $H$, Iseda T, Hino O: Identification of a novel protein (VBP-1) binding to the von Hippel-Lindau (VHL) tumor suppressor gene product. Cancer Res 1996, 56(13):2881-2885.

17. Bharadwaj LA, Davies GF, Xavier IJ, Ovsenek N: L-carnosine and verapamil inhibit hypoxia-induced expression of hypoxia inducible factor (HIF-1 alpha) in H9c2 cardiomyoblasts. Pharmacol Res 2002, 45(3):175-181.

18. Pastorekova S, Zatovicova M, Pastorek J: Cancer-associated carbonic anhydrases and their inhibition. Curr Pharm Des 2008, 14(7):685-698.

19. Svastova E, Zilka N, Zatovicova M, Gibadulinova A, Ciampor F, Pastorek J, Pastorekova S: Carbonic anhydrase IX reduces E-cadherin-mediated adhesion of MDCK cells via interaction with beta-catenin. Exp Cell Res 2003, 290(2):332-345.

20. Svastova E, Witarski W, Csaderova L, Kosik I, Skvarkova L, Hulikova A, Zatovicova M, Barathova M, Kopacek J, Pastorek J, Pastorekova S: Carbonic Anhydrase IX Interacts with Bicarbonate Transporters in Lamellipodia and Increases Cell Migration via Its Catalytic Domain. J Biol Chem 2012, 287(5):3392-3402.

21. Ditte P, Dequiedt F, Svastova E, Hulikova A, Ohradanova-Repic A, Zatovicova M, Csaderova L, Kopacek J, Supuran CT, Pastorekova S, Pastorek J: Phosphorylation of carbonic anhydrase IX controls its ability to mediate extracellular acidification in hypoxic tumors. Cancer Res 2011 71(24):7558-7567.

22. Potter $\mathrm{C}$, Harris AL: Hypoxia inducible carbonic anhydrase IX, marker of tumor hypoxia, survival pathway and therapy target. Cell Cycle 2004 3(2):164-167.

23. Wykoff CC, Beasley NJP, Watson PH, Turner K, Pastorek J, Sibtain A, Wilson GD, Turley H, Talks KL, Maxwell PH, Pugh CW, Ratcliffe PJ, Harris AL: Hypoxia-inducible expression of tumor-associated carbonic anhydrases. Cancer Res 2000, 60(24):7075-7083.

24. Temperini C, Scozzafava A, Puccetti L, Supuran CT: Carbonic anhydrase activators: X-ray crystal structure of the adduct of human isozyme II with L-histidine as a platform for the design of stronger activators. Bioorg Med Chem Lett 2005, 15(23):5136-5141.

25. Zatovicova M, Tarabkova K, Svastova E, Gibadulinova A, Mucha V, Jakubickova L, Biesova Z, Rafajova M, Ortova Gut M, Parkkila S, Parkkila AK, Waheed A, Sly WS, Horak I, Pastorek J, Pastorekova S: Monoclonal antibodies generated in carbonic anhydrase IX-deficient mice recognize different domains of tumour-associated hypoxia-induced carbonic anhydrase IX. J Immunol Methods 2003, 282(1-2):117-134.

26. Svastova E, Hulikova A, Rafajova M, Zatovicova M, Gibadulinova A, Casini A Cecchi A, Scozzafava A, Supuran CT, Pastorek J, Pastorekova S: Hypoxia activates the capacity of tumor associated carbonic anhydrase IX to acidify extracellular pH. FEBS Lett 2004, 577(3):439-445.

27. Chez MG, Buchanan CP, Aimonovitch MC, Becker M, Schaefer K, Black C, Komen J: Double-blind, placebo-controlled study of L-carnosine supplementation in children with autistic spectrum disorders. J Child Neurol 2002, 17(11):833-837.

28. Matsukura T, Takahashi T, Nishimura Y, Ohtani T, Sawada M, Shibata K: Characterization of crystalline L-carnosine Zn(II) complex (Z-103), a novel anti-gastric ulcer agent: tautomeric change of imidazole moiety upon complexation. Chem Pharm Bull (Tokyo) 1990, 38(11):3140-3146.

29. Nicoletti VG, Santoro AM, Grasso G, Vagliasindi LI, Giuffrida ML, Cuppari C, Purrello VS, Stella AM, Rizzarelli E: Carnosine interaction with nitric oxide and astroglial cell protection. J Neurosci Res 2007, 85(10):2239-2245.

30. Zhang $X$, Song $L$, Cheng $X$, Yang Y, Luan B, Jia L, Xu F, Zhang Z: Carnosine pretreatment protects against hypoxia-ischemia brain damage in the neonatal rat model. Eur J Pharmacol 2011, 667(1-3):202-207.

31. Di Paola R, Impellizzeri D, Salinaro AT, Mazzon E, Bellia F, Cavallaro M, Cornelius C, Vecchio G, Calabrese V, Rizzarelli E, Cuzzocrea S: Administration of carnosine in the treatment of acute spinal cord injury. Biochem Pharmacol 2011, 82(10):1478-1489.

32. Cuzzocrea S, Genovese T, Failla M, Vecchio G, Fruciano M, Mazzon E, Di Paola R, Muia C, La Rosa C, Crimi N, Rizzarelli E, Vancheri C: Protective effect of orally administered carnosine on bleomycin-induced lung injury. Am J Physiol Lung Cell Mol Physiol 2007, 292(5):1095-1104.

33. Pfister F, Riedl E, Wang Q, Vom Hagen F, Deinzer M, Harmsen MC, Molema G, Yard B, Feng Y, Hammes HP: Oral carnosine supplementation prevents vascular damage in experimental diabetic retinopathy. Cell Physiol Biochem 2011, 28(1):125-136.

34. Severin SE, Boldyrev AA, Stvolinskiĭ SL, Bordiukov MM, Goncharenko EN, Deev LI, Malinina IE, Kudriashov IB: The radiation modifying properties of carnosine. Radiobiologiia 1990, 30(6):765-768.

35. Guney Y, Turkcu UO, Hicsonmez A, Andrieu MN, Guney HZ, Bilgihan A, Kurtman C: Carnosine may reduce lung injury caused by radiation therapy. Med Hypotheses 2006, 66(5):957-959. 
36. Gardner ML, Illingworth KM, Kelleher J, Wood D: Intestinal absorption of the intact peptide carnosine in man, and comparison with intestinal permeability to lactulose. J Physio/ 1991, 439:411-422.

37. Oppermann H, Meixensberger J, Gaunitz F: HIF Signalling in Glioblastoma under the Influence of Carnosine. Leipzig: 63rd Annual Meeting of the German Society of Neurosurgery (DGNC) Joint Meeting with the Japanese Neurosurgical Society (JNS), 13-16 June; 2012.

38. Chaudary N, Hill RP: Hypoxia and metastasis. Clin Cancer Res 2007, 13(7):1947-1949.

39. Harris AL: Hypoxia - A key regulatory factor in tumour growth Nat Rev Cancer 2002, 2(1):38-47.

40. Pastorekova S, Ratcliffe PJ, Pastorek J: Molecular mechanisms of carbonic anhydrase IX-mediated $\mathrm{pH}$ regulation under hypoxia. BJU Int 2008, 101:8-15.

41. Hipkiss AR, Cartwright SP, Bromley C, Gross SR, Bill RM: Carnosine: can understanding its actions on energy metabolism and protein homeostasis inform its therapeutic potential? Chem Cent J 2013, 7:38.

42. Renner C, Asperger A, Seyffarth A, Meixensberger J, Gebhardt R, Gaunitz F: Carnosine inhibits ATP production in cells from malignant glioma. Neurol Res 2010, 32(1):101-105.

43. Dubois L, Peeters S, Lieuwes NG, Geusens N, Thiry A, Wigfield S, Carta F, McIntyre A, Scozzafava A, Dogne JM, Supuran CT, Harris AL, Masereel B, Lambin P: Specific inhibition of carbonic anhydrase IX activity enhances the in vivo therapeutic effect of tumor irradiation. Radiother Oncol 2011, 99(3):424-431

44. Gaunitz F, Hipkiss AR: Carnosine and cancer: a perspective. Amino Acids 2012, 43(1):135-142.

45. Nagai K, Suda T: Antineoplastic effects of carnosine and beta-alanine physiological considerations of its antineoplastic effects. Nihon Seirigako Zasshi - J Physiol Soc Jpn 1986, 48(11):741-747.

46. Horii Y, Shen J, Fujisaki Y, Yoshida K, Nagai K: Effects of I-carnosine on splenic sympathetic nerve activity and tumor proliferation. Neurosci Lett 2012, 510(1):1-5

47. Chuang CH, Hu ML: L-carnosine inhibits metastasis of SK-Hep-1 cells by inhibition of matrix metaoproteinase- 9 expression and induction of an antimetastatic gene, nm23-H1. Nutr Cancer 2008, 60(4):526-533.

\section{Submit your next manuscript to BioMed Central and take full advantage of:}

- Convenient online submission

- Thorough peer review

- No space constraints or color figure charges

- Immediate publication on acceptance

- Inclusion in PubMed, CAS, Scopus and Google Scholar

- Research which is freely available for redistribution 